\title{
OPTIMIZATION OF LINEAR WIRE ANTENNA ARRAYS TO INCREASE MIMO CAPACITY USING SWARM INTELLIGENCE
}

\author{
U. Olgun, C. A. Tunc, D. Aktas, V. B. Ertürk, and A. Altintas \\ Dept. of Electrical and Electronics Engineering, Bilkent University, Ankara,Turkey, e-mail: celal@ee.bilkent.edu.tr
}

Keywords: MIMO, linear wire antenna arrays, particle swarm optimization, channel capacity, mutual coupling.

\begin{abstract}
Free standing linear arrays (FSLA) are analyzed and optimized to increase MIMO capacity. A MIMO channel model based on electric fields is used. The effects of mutual interactions among the array elements are included into the channel matrix using Method of Moments (MoM) based full-wave solvers. A tool to design an antenna array of superior MIMO capacity for any specified volume is developed. Particle swarm optimization is used as the main engine for the optimization tasks of the tool. Uniform linear arrays, uniform circular arrays and non-uniform arrays are analyzed and compared in terms of their channel capacity.
\end{abstract}

\section{Introduction}

Multiple Input Multiple Output (MIMO) technology has attracted huge attention in wireless communications, due to its ability of offering significant increase in data throughput and link range without additional bandwidth or transmit power in the presence of multi-path scattering. MIMO systems achieve this success by using a number of multi-element antenna arrays both at transmitter and receiver which leads to higher spectral efficiency and link reliability $[1,2]$.

Even though MIMO offers high channel capacity in a limited bandwidth, some work still needs to be done to achieve that. To acquire the highest capacity out of a MIMO channel, the choice of array type and configuration at transmitter (TX) and receiver $(\mathrm{RX})$ is a fundamental design issue. Uniform linear arrays (ULA) are intensely studied for MIMO systems. However the question of whether non-uniform linear arrays are able to outperform ULA in terms of their capacity still remains unanswered.

For antenna engineers, it is desirable to have a tool with the capability of designing antenna arrays of superior channel capacities to be placed in any given arbitrary volume. In order to extract the most from the arbitrary volume, the tool should optimize the parameters of antenna arrays such as the number of elements used, individual lengths and locations of the array elements. Particle Swarm Optimization (PSO), a new yet popular optimization algorithm inspired from the swarm behavior of bees, can be used for this purpose since the algorithm has been proved to be very effective in solving computational optimization problems [3]. In this study, we developed the tool that designs an antenna array composed of freestanding linear wire antennas whose lengths and locations are optimized using PSO for higher MIMO channel capacity.
Although the use of MIMO systems creates an improvement on the performance of wireless systems in terms of channel capacity, it presents its own challenges. Physical limitations in portable devices force multiple antennas to be spaced closely which result in the generation of considerable amount of mutual coupling between antenna elements. The effect of mutual coupling on these systems is significant and can not be neglected [4-15]. Thereof in this study, MIMO capacity is calculated using a channel model with electric fields (MEF) which is proven to be capable of analyzing array characteristics such as electrical and geometrical properties in detail [15]. The MEF is based on a full wave hybrid method of moments (MoM)/Green's function technique that can handle majority of the electromagnetic effects such as mutual coupling among array elements.

Organization of the paper is as follows: The MIMO system model is discussed in Section 2, then the model with electric fields is formulated in Section 3. In Section 4, a brief analysis of PSO outlining its working principle is provided. Numerical results, including array designs optimized by PSO, are given in Section 5 along with the comparisons of non-uniform and uniform linear arrays. Finally, concluding remarks are presented. Throughout this paper, an $e^{j w t}$ time convention is used and suppressed from the expressions.

\section{MIMO System Model}

In this study, we consider a non-line-of-sight (NLoS) channel whose scattering environment is a three dimensional (3D), single bounce geometric model. The model includes a local cluster of scatterers located at far zone and distributed uniformly in a volume of spherical shell around transmitter array. Under the constraint that fading is frequency-flat, the relation between received signal vector, $\bar{v}^{r x}$ and the transmitted one, $\bar{v}^{t x}$ can be written as

$$
\bar{v}^{r x}=\mathbf{H} \bar{v}^{t x}+\bar{n},
$$

where $\bar{n}$ is the additive white Gaussian noise vector with zero mean independent identically distributed (i.i.d.) elements with unit variance. The channel matrix $\mathbf{H}$ in Equation (1) is an $R \times T$ matrix, where $R$ and $T$ are the number of antenna elements in receiver and transmitter arrays, respectively. Assuming channel knowledge only at the receiver side and positing the transmitted power to be allocated equally for each transmit element, the maximum amount of data to be transmitted reliably (i.e., the Shannon capacity) for a fixed channel realization can be evaluated as [1]

$$
\mathcal{C}=\log _{2}\left(\left|\mathbf{I}+\frac{P_{T}}{T} \mathbf{H} \mathbf{H}^{*}\right|\right),
$$

where $\mathbf{I}$ is the $R \times R$ identity matrix, $|$.$| is the matrix determi-$ nant, $P_{T}=E\left[\bar{v}^{t x *} \bar{v}^{t x}\right]$ is the total transmitted power, (.)* and 
$E[$.$] denotes the Hermitian transpose and expectation operations,$ respectively.

\section{MIMO Channel Model}

Since the channel capacity is determined by the radio propagation conditions of MIMO channel, characterization and modeling of MIMO radio channels for different environments are critical issues. Accuracy of the model used in design plays a vital role in the validity of predicted system performance.

For the sake of accuracy, as was done in [15], we use a full-wave channel model with electric fields (MEF) that not only based on the spatial properties of antenna elements but also includes majority of electromagnetic effects. The model can be realized, in other words one by one entries of $\mathbf{H}$ can be obtained, with a superposition based procedure which was developed in [15] and is used in this study.

The procedure starts with the evaluations of $\mathbf{Z}^{t x}$ and $\mathbf{Z}^{r x}$, the MoM utilized mutual interaction matrices of TX and RX arrays, respectively. Following the evaluations, superposition principle is employed on the elements of TX. The elements are activated one by one in order while others remain deactivated $\left(v_{n}^{t x}=\right.$ $\left.1 \mathrm{~V}, v_{k \neq n}^{t x}=0\right)$. For each activation, current vector given below is utilized.

$$
\bar{i}^{t x}=\left(\mathbf{Z}^{t x}+\mathbf{Z}_{S}\right)^{-1} \bar{v}^{t x},
$$

where $\mathbf{Z}_{S}$ is a diagonal matrix, non-zero entries of which are the source impedances of each transmit element. Essentially, the voltage on the activated TX element will generate a current which in turn radiates a field that induces current on the surrounding TX elements, i.e. the elements are said to be mutually coupled. When Equation (3) is evaluated, it can readily be noticed that the induced currents are already in $\bar{i}^{t x}$ yielding us to conclude that mutual coupling and other EM effects are taken into account.

The procedure advances with the evaluations of the incident electric fields on the scatters located at the far zone of TX array. The incident electric field on the $p$ th scatterer due to the $n$th transmitter antenna is given by

$$
E_{n p, \theta_{1}}=j \eta_{0} i_{n}^{t x} \frac{e^{-j k r_{n p}}}{2 \pi r_{n p}} \frac{\cos \left(k h_{n} \cos \theta_{1, p}\right)-\cos k h_{n}}{\sin \theta_{1, p}} .
$$

In Equation (4), $\eta_{0}$ denotes the intrinsic impedance of free space, $i_{n}^{t x}$ stands for the current flowing on the $n$th TX element, $r_{n p}$ is the distance between $n$th TX element and $p$ th scatterer, $k$ is the free space propagation constant, $h_{n}$ is the half length of $n$th TX element and $\theta_{1, p}$ is the elevation angle of the $p$ th scatterer in the spherical coordinate system, whose origin coincides with the center of the TX array. The total incident field on the $p$ th scatterer from TX array is obtained as

$$
E_{p, \theta_{1}}=\sum_{n=1}^{T} E_{n p, \theta_{1}}
$$

Each scatterer is designated to have a scattering coefficient, $\alpha_{p}$, which is an i.i.d. Gaussian random variable with zero mean and unit variance. Assuming each scatterer as an isotropic radiator, the field scattered from the $p$ th scatterer impinging on the $m$ th receiver antenna, $E_{p m, \theta_{2}}$, can be expressed as in follows

$$
E_{p m, \theta_{2}}=\alpha_{p} E_{p, \theta_{2}} \frac{e^{-j k r_{m p}}}{r_{m p}},
$$

where $r_{m p}$ is the distance between $m$ th RX element and $p$ th scatterer. It should be noted that a different spherical coordinate system is chosen in Equation (6). As opposed to $\theta_{1, p}$ in Equation (4), whose origin coincides with the center of the TX array, the origin of $\theta_{2, p}$ coincides with the center of the RX array.

Total field received by the $m$ th receiver element is then formulated and received signal vector, $\bar{v}^{r x}$, is written as follows

$$
\begin{aligned}
& E_{m}=\sum_{p=1}^{S}-\sin \theta_{2, p} E_{p m, \theta_{2}}, \\
& \bar{v}^{r x}=\mathbf{Z}_{L}\left(\mathbf{Z}^{r x}+\mathbf{Z}_{L}\right)^{-1} \bar{v},
\end{aligned}
$$

where $\mathbf{Z}_{L}$ is a diagonal matrix, non-zero entries of which are the load impedances of each receive element and $\bar{v}$ is the open circuit voltage vector obtained from the total received fields on RX elements. The entries of $\bar{v}$ of are evaluated by

$$
v_{m}=E_{m} \sum_{p=1}^{S} 2 \frac{\cos \left(k h_{m} \cos \theta_{2, p}\right)-\cos k h_{m}}{k \sin ^{2} \theta_{2, p}},
$$

where $h_{m}$ is the half length of $m$ th RX element. With $v_{n}^{t x}=1$ and $v_{m}^{r x}$ information at hand, the entries of $\mathbf{H}$ is then readily evaluated as,

$$
h_{m n}=\frac{v_{m}^{r x}}{v_{n}^{t x}}=v_{m}^{r x}, \quad v_{k \neq n}^{t x}=0 .
$$

The procedure proceeds with the activation of next TX element, i.e. Equations (3) to (10) are reevaluated. When the activation processing of all elements are finished, the procedure terminates and we have the full knowledge about the entries of $\mathbf{H}$.

\section{Particle Swarm Optimization}

Particle swarm optimization (PSO) is a stochastic evolutionary computation technique that is inspired by the social behavior of organisms such as bee swarming or bird flocking. In PSO, each particle of the swarm starts from a random location and then flies over the multidimensional search space to look for promising regions according to the experiences of both its own and those of the swarm's. Recently, PSO has been applied to several electromagnetic problems and proved its supreme efficiency in solving complex computational electromagnetic problems [3].

PSO borrows its simplicity and efficiency from the utilization of only one operator, the so-called velocity operator. Each particle has its own velocity vector along with the position, from which its next position is determined. The velocity vector of each particle is updated based on the personal best location (i.e., pbest) as well as the best location for the entire swarm (i.e., gbest), which allows information sharing and cooperation among particles. The position updates for the entire swarm are repeated until convergence is achieved. Velocity and position of a particle at any instant is formulated by the following equations.

$$
\begin{gathered}
x_{n, t+1}=x_{n, t}+v_{n, t} \times \Delta t, \\
v_{n, t+1}=K\left[v_{n, t}+\varphi_{1} \times \mathcal{U}(0,1) \times\left(\text { pbest }_{n, t}-x_{n, t}\right)\right. \\
\left.+\varphi_{2} \times \mathcal{U}(0,1) \times\left(\text { gest }_{n, t}-x_{n, t}\right)\right],
\end{gathered}
$$

where $x_{n, t}$ and $v_{n, t}$ are the particle's position and velocity in $n$th dimension at instant $t, \Delta t$ is the time step which is chosen to be one, $K$ is the constriction factor, $\mathcal{U}(0,1)$ denotes the uniformly 


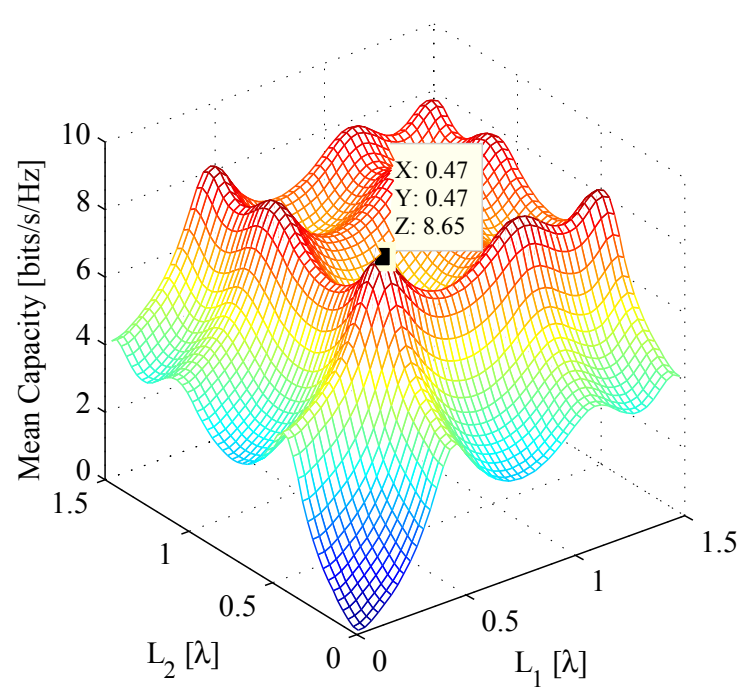

(a) Numerical Solution

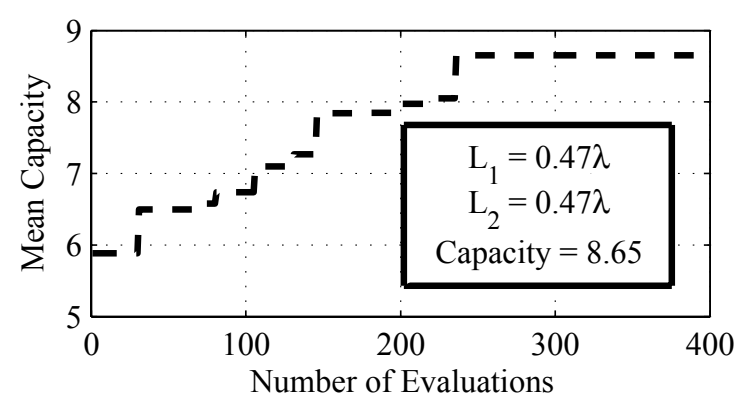

(b) PSO Solution

Figure 1. Solutions for 2 Antennas with a fixed seperation of $0.61 \lambda$.

distributed random numbers between zero and one, finally $\varphi_{1}$ and $\varphi_{2}$ are the scaling factors that determine the relative pull of pbest and gbest of the particles, respectively. As stated and analyzed in [3], the optimal selection of constants mentioned above necessitates the choice of $K$ to be $0.729, \varphi_{1}$ to be 2.8 and $\varphi_{2}$ to be 1.3 , thus removing the need for setting a $v_{\max }$.

Occasionally, particles pass beyond the boundaries of given solution space, hence adoption of a boundary policy to the algorithm is essential. In order to enforce particles to search inside the solution space of interest, several boundary conditions (e.g., reflecting, absorbing, invisible) have been described and analyzed in [16]. Among those examined, damping wall technique suits our application best and therefore is used in this study. In damping wall technique, when a particle attempts to search outside the allowable solution space in one of the dimensions, it is relocated at the boundary of the solution space and the velocity component in that dimension is changed in the opposite direction and multiplied with a random factor between zero and one.

\section{Numerical Results}

Transmitter antenna arrays with different number of elements are designed to be placed in a cube which is centered at the origin, with edges parallel to the axes and with an edge length equal to $\lambda$, free-space wavelength. Throughout this study, dimensions of the volumes used are discretized by $0.01 \lambda$. The designs are made in

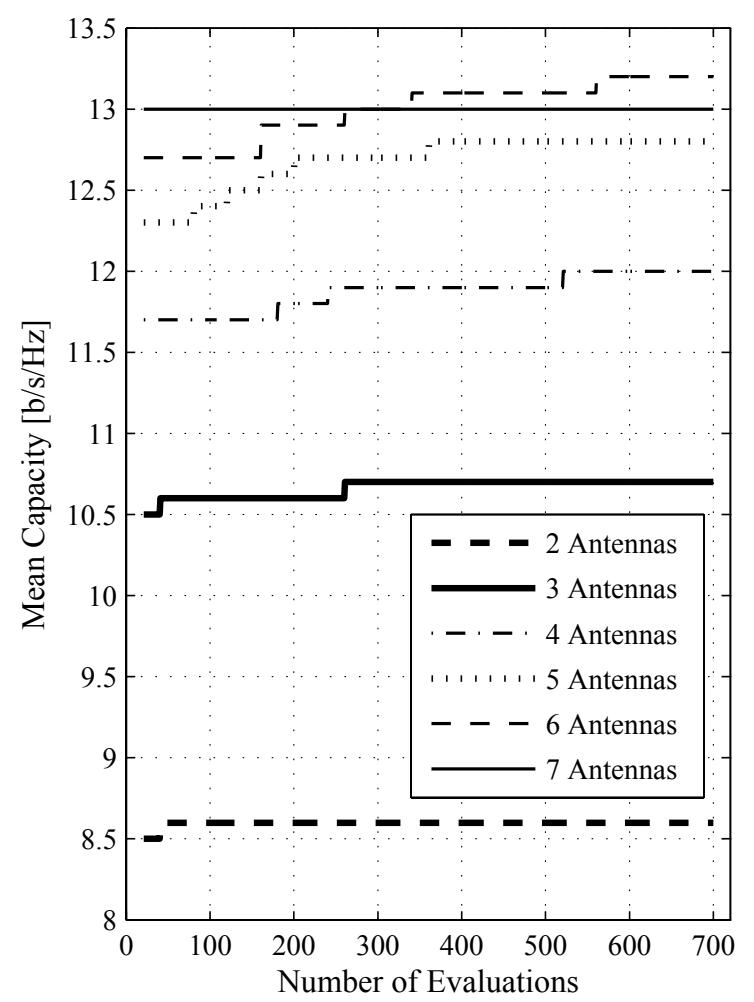

Figure 2. Capacity Improvement over UCA for 2D PSO Optimization.

two phases such that in the first phase, the number of linear antennas to be used and their locations are optimized; whereas in the second phase, their lengths along with those in the first phase are optimized for higher MIMO channel capacity. On the other hand, the receiver array is assumed to be an FSLA located $300 \lambda$ away from the transmitter in a broadside manner, formed by $R=10$ uniform linear dipoles each of which is separated by a distance of $\lambda / 2$. Elements of both TX and RX array have a radius of $\lambda / 200$ and are connected to $50 \Omega$ source and load impedances, respectively. Transmit power of the array is assumed to be $90 \mathrm{~dB}$. The channel is modeled by locating $S=100$ uniformly distributed scatterers around the transmitter within a spherical shell with far zone inner and outer radii and the mean capacity results are obtained by averaging the MIMO channel capacity over $N_{R}=1000$ channel realizations. It should be noted that, $N_{R} \cdot S$ scatterer locations and coefficients are generated and kept in the memory as a "scenario", then used for all numerical simulations. Therefore, effects of these random parameters on comparisons are eliminated for the same scatterer geometry parameters.

As the technique we use in optimization is basically a random process, different mean capacity values can be obtained with a change in the volume used or with the regeneration of scatterers. Therefore in our optimization procedure, we need to take into account the confidence interval of the mean capacity results. To compute the confidence interval, we fixed the array configurations at RX and TX and generated 1000 different "scenario"s each of which has $N_{R} \cdot S$ scatterers. The mean capacities obtained from the aforementioned realizations are observed to have normal distribution. The confidence interval for normal distribution is given as,

$$
\mathcal{C}_{i}=\sigma \sqrt{2} \operatorname{erf}^{-1}(\mathcal{P}),
$$

where $\mathcal{C}_{i}$ is the confidence interval, $\mathcal{P}$ is the level of confidence 


\begin{tabular}{|c||c|c|c|c|c|c|}
\hline$T$ & 2 & 3 & 4 & 5 & 6 & 7 \\
\hline \hline $\mathcal{C}_{u l a}$ & 8.4 & 10.0 & 10.5 & 9.5 & 8.5 & 7.7 \\
\hline $\mathcal{C}_{u c a}$ & 8.5 & 10.5 & 11.7 & 12.3 & 12.7 & 13.0 \\
\hline $\mathcal{C}_{p s o}$ & 8.6 & 10.7 & 12.0 & 12.8 & 13.2 & 13.0 \\
\hline$x_{1}, y_{1}$ & $(0.42,0.10)$ & $(0.44,0.08)$ & $(0.39,-0.04)$ & $(0.34,-0.01)$ & $(0.39,-0.01)$ & $(0.50,0.00)$ \\
\hline$x_{2}, y_{2}$ & $(0.45,-0.50)$ & $(0.50,-0.50)$ & $(0.50,0.50)$ & $(0.50,0.50)$ & $(0.50,0.46)$ & $(0.31,0.39)$ \\
\hline$x_{3}, y_{3}$ & - & $(-0.20,0.50)$ & $(-0.33,-0.50)$ & $(-0.35,0.50)$ & $(-0.38,0.48)$ & $(-0.11,0.49)$ \\
\hline$x_{4}, y_{4}$ & - & - & $(0.50,-0.50)$ & $(-0.38,-0.41)$ & $(-0.26,0.00)$ & $(-0.45,0.22)$ \\
\hline$x_{5}, y_{5}$ & - & - & - & $(0.50,-0.50)$ & $(-0.40,-0.50)$ & $(-0.45,-0.22)$ \\
\hline$x_{6}, y_{6}$ & - & - & - & - & $(0.50,-0.50)$ & $(-0.11,-0.49)$ \\
\hline$x_{7}, y_{7}$ & - & - & - & - & - & $(0.31,-0.39)$ \\
\hline
\end{tabular}

Table 1. Optimum TX Locations for 2D PSO optimization.

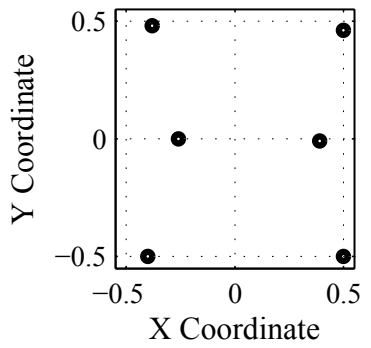

(a) 6 Antennas

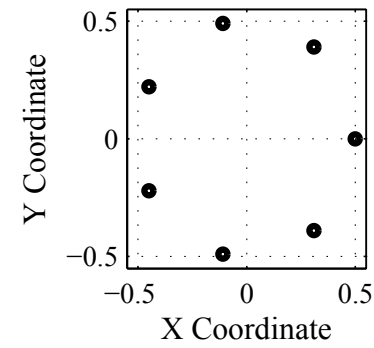

(b) 7 Antennas

Figure 3. Top view geometries of sample designs made by $2 D$ PSO.

given between zero and one and $\sigma$ is the standard deviation of the distribution. For a confidence level of $90 \%$, the confidence interval of our technique is calculated to be 0.05 . As a consequence, even for the most pessimistic case, we may regard an increase of $0.1 \mathrm{bits} / \mathrm{s} / \mathrm{Hz}$ or higher in capacity as an improvement of new design over the existing one.

In order to check the reliability of our PSO implementation, we solve a two element fixed seperation (i.e., $0.61 \lambda$ ) varying length (i.e., from 0.01 to $1.5 \lambda$ ) antenna array both numerically and by PSO for our volume and receiver configuration. The numerical result is presented in Figure 1(a) which depicts that the highest capacity is achieved when lengths of elements are equal to $0.47 \lambda$, which coincides with the PSO results in Figure 1(b). It should be noted that the numerical solution required 22500 cost function evaluations whereas PSO completed it in less than 250 evaluations. By the way, with this numerical solution at hand, we have found the answer to the question whether ULA can be outperformed by non-uniform linear arrays. Figure 1(a) indicates different uniform and non-uniform array configurations outperforming the conventional $0.5 \lambda$ length ULA in terms of MIMO capacity when $T$ is equal to two.

In antenna engineering, uniform circular arrays (UCA) are among the most popular solutions of antenna design problems. As a first step of this study, we seek improvement over the circularity of uniform arrays. In other words, we initialize one of our bees with a UCA of element length $0.5 \lambda$ and employ the PSO to find a better solution than UCA. Since we are looking for an improvement over circularity in this stage of our work, we fix the element lengths to $0.5 \lambda$, i.e. PSO only varies the positions of array elements in a $2 \mathrm{D}$ space which is in fact a square of area $\lambda^{2}$. Optimum channel capacities found by PSO and the phase center locations yielding them are tabulated in Table 1 and improvement over UCA is plotted in Figure 2.

\begin{tabular}{|c|c|c|c|c|c|c|}
\hline$T$ & $\mathcal{C}_{u c a}$ & $\mathcal{C}_{3 D}$ & $x(\lambda)$ & $y(\lambda)$ & $z(\lambda)$ & $\overline{L(\lambda)}$ \\
\hline \multirow{2}{*}{2} & \multirow{2}{*}{8.5} & \multirow{2}{*}{9.0} & $\overline{0.45}$ & 0.47 & -0.265 & 0.47 \\
\hline & & & -0.5 & -0.15 & 0.265 & 0.47 \\
\hline \multirow{3}{*}{3} & \multirow{3}{*}{10.5} & \multirow{3}{*}{11.3} & 0.27 & -0.05 & 0.27 & 0.46 \\
\hline & & & 0.15 & 0.5 & -0.265 & 0.47 \\
\hline & & & -0.32 & -0.5 & 0.22 & 0.46 \\
\hline \multirow{4}{*}{4} & \multirow{4}{*}{11.7} & \multirow{4}{*}{12.7} & 0.31 & -0.07 & 0.01 & 0.46 \\
\hline & & & 0.26 & 0.5 & -0.265 & 0.47 \\
\hline & & & -0.42 & -0.5 & 0.4 & 0.46 \\
\hline & & & 0.5 & -0.5 & 0.27 & 0.46 \\
\hline \multirow{5}{*}{5} & \multirow{5}{*}{12.3} & \multirow{5}{*}{13.7} & 0.37 & -0.13 & 0.265 & 0.47 \\
\hline & & & 0.31 & 0.5 & 0.05 & 0.46 \\
\hline & & & -0.5 & 0.5 & -0.09 & 0.46 \\
\hline & & & -0.39 & -0.5 & -0.05 & 0.46 \\
\hline & & & 0.5 & -0.5 & 0.03 & 0.46 \\
\hline \multirow{6}{*}{6} & \multirow{6}{*}{12.7} & \multirow{6}{*}{14.0} & 0.48 & 0.3 & -0.035 & 0.47 \\
\hline & & & 0.5 & 0.5 & 0 & 0.46 \\
\hline & & & -0.33 & 0.48 & 0.06 & 0.46 \\
\hline & & & -0.28 & 0.04 & 0.05 & 0.46 \\
\hline & & & -0.5 & -0.5 & 0.005 & 0.47 \\
\hline & & & 0.48 & -0.5 & -0.005 & 0.47 \\
\hline \multirow{7}{*}{7} & \multirow{7}{*}{13.0} & \multirow{7}{*}{14.3} & 0.44 & -0.07 & -0.02 & 0.46 \\
\hline & & & 0.44 & 0.46 & 0 & 0.46 \\
\hline & & & -0.37 & 0.5 & -0.005 & 0.45 \\
\hline & & & -0.42 & 0.14 & 0.005 & 0.45 \\
\hline & & & -0.49 & -0.28 & -0.27 & 0.46 \\
\hline & & & -0.47 & -0.5 & 0.04 & 0.46 \\
\hline & & & 0.44 & -0.49 & -0.05 & 0.46 \\
\hline
\end{tabular}

Table 2. Optimum TX Locations for 3D PSO optimization

Table 1 depicts the mean capacity (in bits/s/Hz) obtained from the ULA, UCA and the one obtained from PSO along with the locations of antennas (in terms of wavelength) that PSO found as the best on a $x y$-plane of $100^{2}$ grid points. As is obvious from the table, for the plane we used, PSO can find better results than UCA provided that $T$ does not exceed six. However if it is more than that, PSO agrees that UCA is the best solution. Figure 3(a) shows the geometry, which is not a circular one, of the six element TX designed by PSO and Figure 3(b) shows the geometry, which is a circular one, of the seven element TX which is also designed by PSO.

As just mentioned and also illustrated in Figure 2, in the geometry we consider, PSO cannot improve UCA confidently when TX is a seven or more element antenna array. This is the case since with the increase in the number of TX elements used, due to physical limitations, elements are forced to be placed closely which results in the generation of considerable amount of mutual 


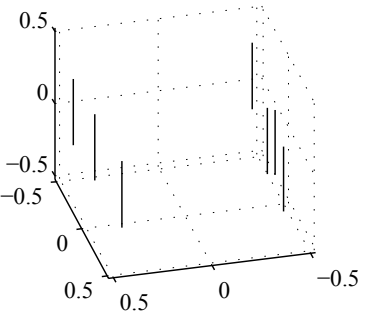

(a) $3 \mathrm{D}$ view

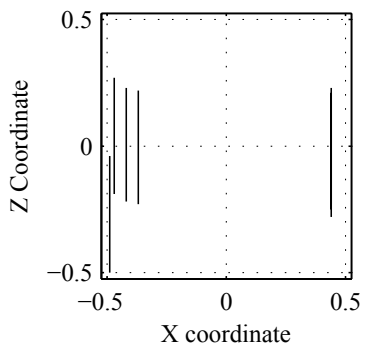

(c) $x z$-view

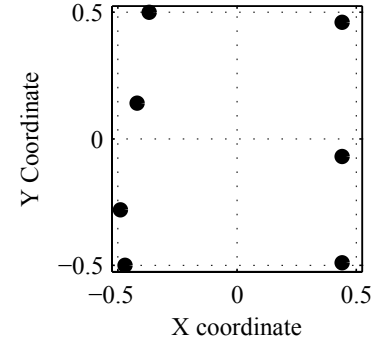

(b) Top view

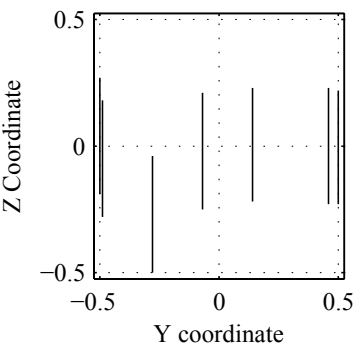

(d) $y z$-view
Figure 4. Geometry of 7 element TX designed by 3D PSO.

coupling. Therefore to achieve the optimum interelement spacing, elements of the TX array are spaced in a circular fashion when $T$ exceeds six. Improvements achieved by PSO over UCA is visualized in Figure 2 which tells us that the mean capacity is improved by $0.1,0.2,0.3,0.5$ and 0.5 all in bits $/ \mathrm{s} / \mathrm{Hz}$ for two, three, four, five and six TX elements, respectively. This yields us to conclude that although there are improvements over circularity of uniform arrays, UCA is still a good design option since improvements are comparatively small for this $\lambda^{2}$ geometry.

By the way, it is crucial to note that by initializing one of our bees with UCA, we have made a smart start. When this is not the case, in other words when we have all our bees initialized randomly, we are obliged to do thousands of more evaluations than we do now since we observe that bees first converge to UCA and then try to improve it. The benefit of using this smart initialization is its fast and certain convergence to the design with optimum channel capacity in relatively low number of evaluations. Figure 2 explains the "relatively low" such that the designs are made in less than 700 evaluations for a solution space of order $100^{2 T}$, i.e. $O\left(100^{2 T}\right)$, where each solution dimension (i.e., $x$ and $y$ which are the phase center coordinates) has a size of 100 per each TX element.

Thereafter, we modify the PSO so as to include the lengths of TX elements into its variable basket. In other words, PSO is now employed to make a 3D design in the cube mentioned at the beginning of this section. Our modified PSO is now powered with the capability of analyzing arrays in nonstaggered, staggered and collinear arrangement. In order to sustain our smart tradition, we initialize one of our particles with the final designs made by PSO in 2D space while another one still starts with a UCA. Remaining particles are released randomly to the solution space of $O\left(100^{4 T}\right)$ where each solution dimension (i.e., $x, y, z$ and $L$ where the first three are the phase center coordinates and $L$ is the length) has a size of 100 per each TX element.

Table 2 reviews the mean capacity obtained from the UCA and introduces the one obtained from 3D PSO along with the optimum phase center location and length for each element in the array. 3D

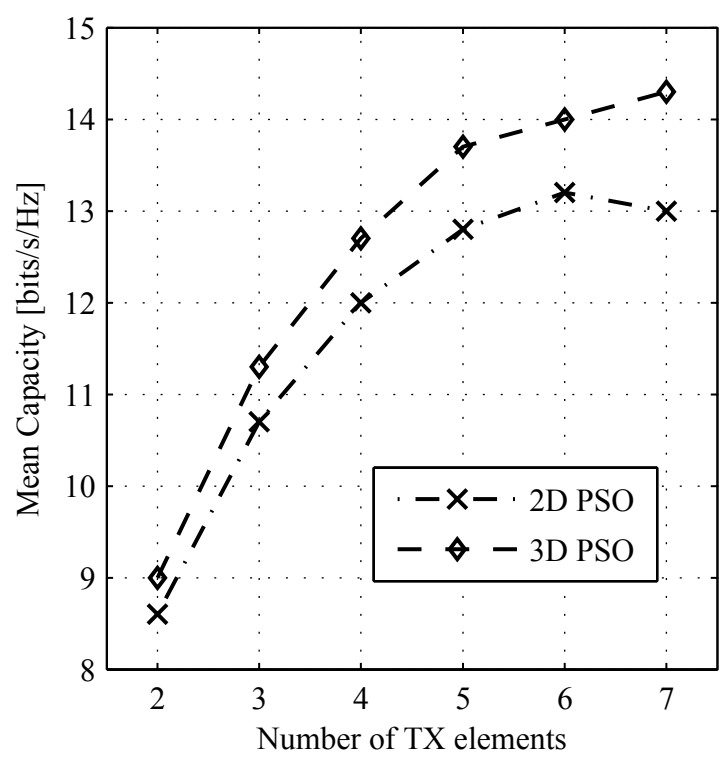

Figure 5. Capacity of $2 D$ and $3 D P S O$ versus number of TX elements used.

PSO changed the $x y$ locations of phase centers of its $2 \mathrm{D}$ counterpart as well as changing the element lengths from $0.5 \lambda$ to $0.47 \lambda$. This results in a capacity increase of $0.4,0.6,0.9$, and 0.8 all in bits/s/Hz over 2D PSO for two, three, four, five and six TX elements, respectively. Noting that the 2D PSO is already an improvement over UCA, it can be concluded that 3D PSO has found significantly better results compared to the ones by UCA and definitely much better than the ULA.

As mentioned in 2D PSO, we are unable to find a better solution than UCA for seven element TX. On the other hand, the design by 3D PSO has managed to outperform UCA because of its ability to place elements in staggered or collinear arrangements. Figure 4 depicts the design made by PSO for seven element TX where elements are seen to be arranged as mentioned before. It should be noted for the Figure 4(c) that since the $x$ coordinate of the three elements' phase centers are the same, it is seen as if there are five elements placed although there are seven. Figure 5 shows the mean capacity for 2D and 3D PSO designs as $T$ varies which also indicates the importance of how TX elements are arranged especially when we use more elements.

\section{Conclusion}

MIMO performance of free standing linear wire antenna arrays, both with uniform and non-uniform elements, are investigated in terms of mean channel capacity. It has been observed that different uniform and non-uniform array configurations outperform the conventional $0.5 \lambda$ length ULA in terms of MIMO capacity.

Since the physical limitations of the volume of interest is one of the main constraints of antenna synthesis, finding optimum locations yielding high data rates is a significant issue for MIMO array design. For this purpose, we use particle swarm optimization (PSO) that is, to the best of our knowledge, introduced to MIMO optimization problems for the first time. We have tested the PSO's handling capability of MIMO problems by solving a predetermined problem numerically and solving the same problem using PSO. PSO has proved its supreme efficiency in solving 
EM problems by finding the optimum antenna geometry in a fast and accurate manner.

A tool that can design antenna arrays of superior MIMO capacity for any given arbitrary volume is developed. The tool is robust and reliable since it uses a full-wave electromagnetic channel model with electric fields to calculate MIMO capacity. In other words in its modeling of MIMO channel, our tool takes majority of EM effects into consideration such as mutual coupling. For a sample volume, using our tool, antenna arrays with optimum data rates are designed. We observe that uniform circular arrays yields considerably good results when the volume used is relatively small.

\section{Acknowledgments}

This work has been supported by the Turkish Scientific and Technical Research Agency (TÜBİTAK) under Grants EEEAG106E081, EEEAG-104E044 and EEEAG-105E065.

\section{References}

[1] I. E. Telatar, "Capacity of multi-antenna Gaussian channels," Europ. Trans. Telecommun., vol. 10, pp. 585-595, Nov. 1999.

[2] G. J. Foschini and M. J. Gans, "On limits of wireless communications in a fading environment when using multiple antennas," Wireless Personal Commun., vol. 6, pp. 311-335, Mar. 1998.

[3] J. Robinson and Y. Rahmat-Samii, "Particle swarm optimization in electromagnetics," IEEE Trans. Antennas Propag., vol. 52, pp. 397-407, Feb. 2004.

[4] R. Janaswamy, "Effect of element mutual coupling on the capacity of fixed length linear arrays," IEEE Antennas Wireless Propagat. Lett., vol. 1, no. 1, pp. 157-160, 2002.

[5] T. Svantesson and A. Ranheim, "Mutual coupling effects on the capacity of multielement antenna systems," in Proc. IEEE Int. Conf. Acoustics, Speech, and Signal Processing (ICASSP'01), Salt Lake City, UT, May 2001, pp. 2485-2488.

[6] J. Andersen and B. K. Lau, "On closely coupled dipoles in a random field," IEEE Antennas Wireless Propagat. Lett., vol. 5, no. 1, pp. 73-75, 2006.

[7] B. K. Lau, J. B. Andersen, G. Kristensson, and A. F. Molisch, "Impact of matching network on bandwidth of compact antenna arrays," IEEE Trans. Antennas Propagat., vol. 54, no. 11 , pp. 3225-3238, 2006.

[8] M. A. Jensen and B. Booth, "Optimal uncoupled impedance matching for coupled MIMO arrays," in The first European Conference on Antennas and Propagation (EuCAP 2006), Nice, France, Nov. 2006.

[9] J. W. Wallace and M. A. Jensen, "Mutual coupling in MIMO wireless systems: A rigorous network theory analysis," IEEE Trans. Wireless Commun., vol. 3, no. 4, pp. 1317-1325, July 2004.

[10] — " "Termination-dependent diversity performance of coupled antennas: Network theory analysis," IEEE Trans. Antennas Propagat., vol. 52, no. 1, pp. 98-105, Jan. 2004.

[11] C. A. Tunc, D. Aktas, and A. Altintas, "On arrays of dipole antennas with mutual coupling in MIMO wireless channels," in The 15th IST Mobile \& Wireless Communication Summit, Mykonos, Greece, June 2006.
[12] _ "Performance analysis of uniform circular arrays with mutual coupling in MIMO channels," in the 2006 IEEE International Symposium on Antennas and Propagation and USNC/URSI National Radio Science Meeting, Albuquerque, NM, USA, July 2006.

[13] C. A. Tunc, E. Irci, O. Bakir, D. Aktas, V. B. Ertürk, and A. Altintas, "Investigation of planar and conformal printed arrays for MIMO performance analysis," in The first European Conference on Antennas and Propagation (EuCAP 2006), Nice, France, Nov. 2006.

[14] _ "A model with electric fields for the inclusion of mutual coupling effects in the MIMO channel," in The 2007 IEEE International Symposium on Antennas and Propagation, Honolulu, HI, USA, June 2007.

[15] — _A MIMO channel model with electric fields," IEEE Trans. Antennas Propag., submitted for publication.

[16] S. Xu and Y. Rahmat-Samii, "Boundary conditions in particle swarm optimization revisited," IEEE Trans. Antennas Propag., vol. 55, Mar. 2007. 